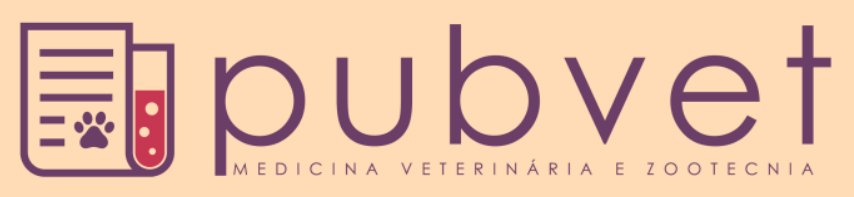

https://doi.org/10.22256/pubvet.v12n4a75.1-6

\title{
Análise do conforto térmico e sua influência na produção e qualidade do leite em ambiente de domínio de cerrado
}

\author{
Oscar Vitor Neto ${ }^{\ominus}$, Dyb Youssef Bittar ${ }^{\ominus} 2 *$ \\ ${ }^{1}$ Aluno do Curso de Agronomia, Faculdade Unievangélica de Goianésia, Brasil. \\ ${ }^{2}$ Docente do Curso de Agronomia, Unievangélica de Goianésia, Brasil \\ *Autor para correspondência, E-mail: dybittar@hotmail.com
}

\begin{abstract}
RESUMO. São muitos os fatores que contribuem para o aumento dos efeitos do estresse térmico como, produtividade, raça e sistema de criação. Neste sentido objetivou se com este trabalho avaliar os reflexos do estresse térmico, sobre a produção e composição do leite nos meses de maio a setembro de 2017, todos esses dados foram coletados logo a após a ordenha de forma não invasiva ao bem-estar do animal. Os dados foram coletados através do uso de termômetro digital e o exame de leite para composição do leite realizado no Laboratório de qualidade de leite (LQL) da Universidade Federal de Goiás (UFG) em Goiânia. Após tabulação dos dados foi comparado os tratamentos com os teores de sólidos encontrados no leite e produtividade das matrizes que fizeram parte do experimento, onde não foi encontrado diferença significativa nos tratamentos avaliados, mostrando que a promoção do bem-estar animal e de extrema importância para aumento e qualidade da produção do leite.
\end{abstract}

Palavras chave: Bovinos, leite, estresse térmico, sólidos, qualidade

\section{Thermal comfort analysis and its influence on the production and quality of milk in the cerrado domain environment}

\begin{abstract}
There are many factors that contribute to the increased effects of thermal stress such as productivity, breed and breeding system. In this sense, the purpose of this study was to evaluate the effects of thermal stress on milk production and composition, from May to September 2017, all of which were collected after milking at in a non-invasive way to the welfare of the animal. The data were collected through the use of a digital thermometer and the milk test for milk composition performed at the Milk Quality Laboratory (LQL) of the Federal University of Goiás (UFG) in Goiânia. After tabulation of the data, the treatments with the contents of solids found in the milk and productivity of the matrices that were part of the experiment were compared, where no significant difference was found in the evaluated treatments, showing that the promotion of animal welfare and of extreme importance for increase and quality of milk production.
\end{abstract}

Keywords: Cattle, milk, heat stress, solids, quality

\section{Análisis del conforto térmico y su influencia en la producción y calidad de la leche en ambiente de dominio de cerrado}

RESUMEN. Son muchos los factores que contribuyen al aumento de los efectos del estrés térmico como, productividad, raza y sistema de cría. En este sentido se objetivó con este trabajo evaluar los reflejos del estrés térmico, en la producción y composición de la leche en los meses de mayo a septiembre de 2017, todos esos datos fueron recolectados después 
del ordeño de forma no invasiva al bienestar del animal. Los datos fueron recolectados a través del uso de termómetro digital y el examen de leche para composición de la leche realizada en el Laboratorio de calidad de leche (LQL) de la Universidad Federal de Goiás (UFG) en Goiânia. Después de la tabulación de los datos se compararon los tratamientos con los niveles de sólidos encontrados en la leche y la productividad de las matrices que formaron parte del experimento, donde no se encontró diferencia significativa en los tratamientos evaluados, mostrando que la promoción del bienestar animal y de extrema importancia para el aumento y la calidad de la producción de leche.

Palabra clave: Bovinos, leche, estrés térmico, sólidos, calidad

\section{Introdução}

A pecuária leiteira a bastante tempo vem trabalhando em pesquisas analisando o efeito da temperatura ambiente no sentido de melhorar a produção. Por estarmos em um país tropical com temperaturas médias do ar entre $20^{\circ} \mathrm{C} \mathrm{e} 32^{\circ} \mathrm{C}$ em boa parte do ano, chegando a temperaturas de $35^{\circ} \mathrm{C}$ a $38^{\circ} \mathrm{C}$, podemos dizer que esse é um dos principais desafios para determinados sistemas da atividade leiteira, sabendo que estas temperaturas são altas para bovinos especializados em produção de leite (Rezende et al., 2016).

Head (1996) e Rodrigues et al. (2010) descrevem a matriz leiteira bovina como um animal homeotérmico, com temperatura interna média de $38,5^{\circ} \mathrm{C}$, frequência cardíaca de 60 a 80 pulsações por minuto e 10 a 30 movimentos por minuto de frequência respiratória. A temperatura corporal do animal pode sofrer pequenas variações durante o dia, sendo mais alta no final das tardes e no início da noite, variando também durante o ciclo estral e entre as estações do ano.

Craesmeyer et al. (2017) relatam que a atividade de pastoreio de matrizes leiteiras em áreas sombreadas, foi 2,5 vezes maior do que em áreas sob o sol pleno. Em sistemas extensivos a oferta de pastagem com disponibilidade de água, áreas com sombra e abrigos auxiliam no controle da temperatura corpórea e resultam em uma combinação de fatores que interferem na saúde animal, desempenho e comportamento geral de maneira benéfica.

Segundo Rezende et al. (2016) a temperatura retal é um fator que nos auxilia para determinação do equilíbrio calórico e que pode ser usado na avaliação do estresse calórico. As oscilações de temperatura retal podem ser afetadas por fatores extrínsecos (hora do dia, ingestão de alimentos e de água, velocidade do vento, estação do ano) e também por fatores intrínsecos (idade, raça e estado fisiológico). Dependendo dos horários do dia a temperatura retal pode ser influenciada em função disso, animais que apresentam alta atividade durante $\mathrm{o}$ dia tem variação na temperatura retal considerando a manhã e à tarde sendo mínima e máxima respectivamente.

Alguns autores como Rezende et al. (2016) avaliando as interferências do estresse térmico em vacas leiteiras já realizaram trabalhos semelhantes, onde observaram que em diferentes horas do dia da manhã ou da tarde tem maior ou menor temperatura retal e que isso nos mostra o momento em que o animal tem maior ou menor estresse térmico e que no verão em função de maior umidade relativa a temperatura corporal dos animais aumenta o que pode contribuir com o aumento da temperatura uterina, responsável pela redução da taxa de concepção.

O objetivo do trabalho foi mostrar que mesmo em condições de altas temperatura é possível viabilizar a produção de leite minimizando os efeitos do estresse térmico pelo manejo e fornecimento de áreas com sombras e água de qualidade em abundância e de fácil acesso para os animais.

\section{Material e Métodos}

$\mathrm{O}$ experimento foi conduzido no período de maio a setembro de 2017, na Fazenda Vera Cruz do Grupo Otavio Lage localizada no município de Goianésia - GO, mesorregião do Centro Goiano e caracterizada pelas seguintes coordenadas cartesianas Latitude sul $15.31^{\circ}$ e longitude oeste $49.12^{\circ}$, altitude aproximada de $640 \mathrm{~m}$. A região do experimento apresenta clima AW que significa clima tropical com estação seca no inverno com temperatura média de $24,4^{\circ} \mathrm{C}$ e pluviosidade média anual de $1502 \mathrm{~mm}$ (Köppen \& Geiger, 1928). A tipologia climática tropical se faz presente na maior parte do estado, apresentando invernos secos e verões chuvosos.

Foram utilizadas matrizes bovinas mestiças (Holandês x zebu) lactantes, entre 40 a 60 dias de paridas e apresentando produção de leite em torno 
de $18 \mathrm{~kg} \mathrm{dia}^{-1}$. Os animais foram divididos em quatro grupos, de acordo com a temperatura retal (TR) de até $38^{\circ} \mathrm{C}$, de $38,1^{\circ} \mathrm{C}$ a $38,5^{\circ} \mathrm{C}$, de $38,6^{\circ}$ $\mathrm{C}$ a $39^{\circ} \mathrm{C}$ e $39,1^{\circ} \mathrm{C}$ a $39,5^{\circ} \mathrm{C}$.

A temperatura dos animais foi monitorada e coletada no período da manhã (07:00 h) e a tarde $(17: 00$ h) e logo após foi feita uma média para determinação da temperatura retal. A medição foi feita com a utilização de termômetro digital inserido no reto do animal, já a temperatura do ambiente foi coletada no site do Instituto nacional de Meteorologia (INMET, 2017). A coleta dos dados de contagem de células somáticas (CCS) e composição do leite é feito mensalmente e individualmente, em que, o leite é coletado e enviado ao laboratório LQL da UFG em Goiânia e em seguida é emitido um laudo do exame com os dados.

As vacas mestiças pastejavam em piquetes, $o$ fornecimento de silagem de resíduo de milho, foi realizado em cocho de concreto, localizados próximo a sala de ordenha. A ordenha foi realizada mecanicamente, duas vezes ao dia, às 05:40 h e às 15:00 h, em sala de ordenha mecânica, coberto com telhas de zinco, com pé direito de $5 \mathrm{~m}$, composto por um corredor central e postos de ordenha coletivo para 10 animais, sendo fechado lateralmente com canos metálicos para facilitar a circulação de ar. Os animais não recebem concentrado durante a ordenha. $\mathrm{O}$ piso era de cimento rugoso, com declividade de aproximadamente $1,5 \%$ para facilitar o escoamento dos dejetos. Durante todo o período de coleta de dados todas as vacas, independentemente do nível produtivo, recebiam $2,0 \mathrm{~kg}$ de concentrado pela manhã e $2,0 \mathrm{~kg}$ à tarde misturados homogeneamente na silagem. A média de produção foi feita com base em duas pesagens diárias durante todo o período do experimento. As variáveis analisadas nos diferentes tratamentos foram: produção mensal de leite, composição do leite, sendo CCS, proteína bruta (PB) e gordura. Os tratamentos foram, temperatura retal (TR) $\left(38^{\circ}\right.$ $\mathrm{C}, 38,5^{\circ} \mathrm{C}, 39^{\circ} \mathrm{C}$ e $39,5^{\circ} \mathrm{C}$ ), as variáveis $\mathrm{CCS}, \mathrm{PB}$ e gordura com 5 repetições (de acordo com os meses, maio, junho, julho, agosto e setembro) foram submetidos ao delineamento inteiramente casualizado. Os dados foram submetidos a ANOVA. As médias foram comparadas, utilizando o teste Tukey, a 5\% de probabilidade.

\section{Resultados e discussão}

$\mathrm{O}$ estresse térmico pode ser amenizado pelo manejo e adoção de práticas que favorecem a redução do mesmo, como construção de sombras e fornecimento de água sem restrição e de fácil acesso. Como prova gráfico 1 demonstra que a CCS reduz mesmo tendo a temperatura média diária acima do recomendado. Da mesma forma, ocorre para proteína (Gráfico 2), gordura (Gráfico 3) e produção de leite (Gráfico 4).

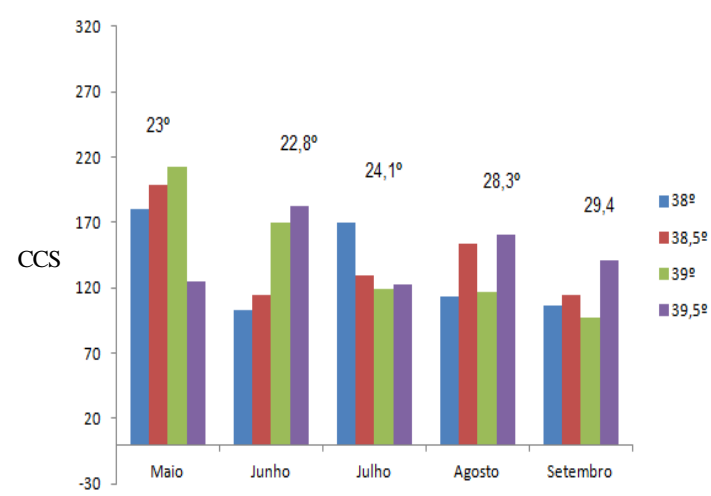

Gráfico 1. Relação da CCS com a temperatura ambiente em diferentes temperaturas retais.

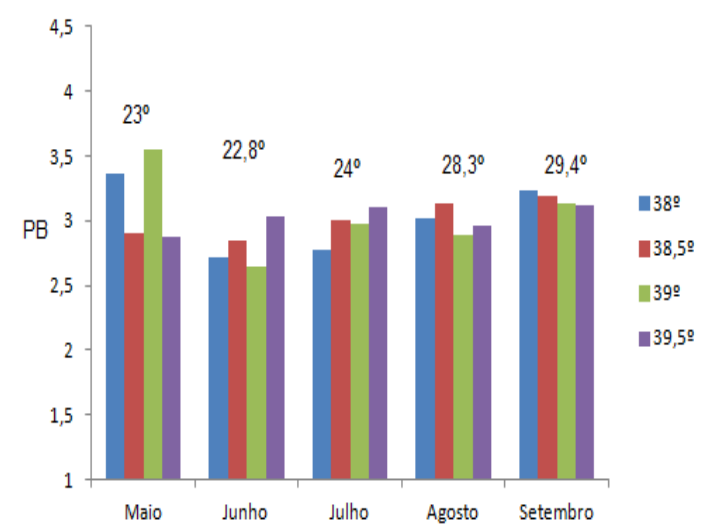

Gráfico 2. Relação da proteína bruta com a temperatura ambiente em diferentes temperaturas retais.

Como os animais são especializadas na produção de leite e alta eficiência na conversão dos alimentos, os animais de alta produção apresentam metabolismo acelerado e alta produção de calor metabólico, tornando assim mais suscetível ao estresse térmico. Devido ao seu efeito sobre o consumo alimentar, o estresse térmico acomete o metabolismo da glândula mamária e da composição do leite (Arcaro Junior et al., 2003). 


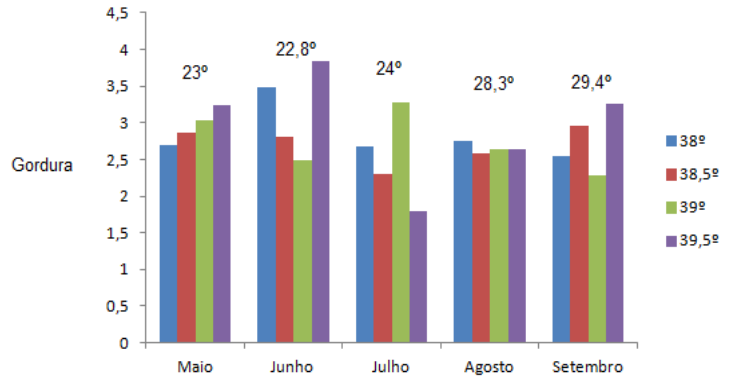

Gráfico 3. Relação da gordura com a temperatura ambiente em diferentes temperaturas retais.

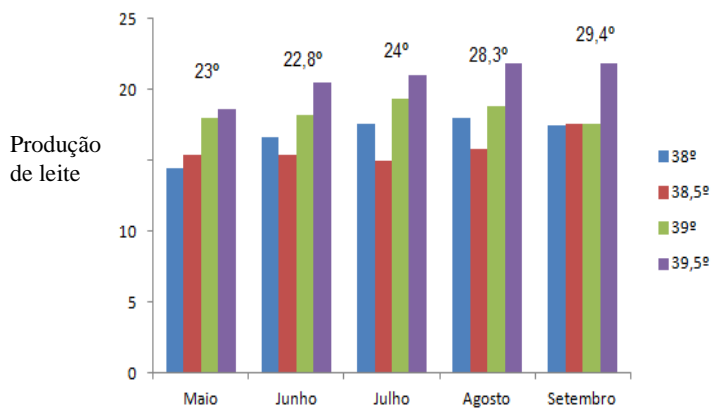

Gráfico 4. Relação da produção média mensal com a temperatura ambiente em diferentes temperaturas retais.

Segundo Pires \& Campos (2004), animais que apresentam TR entre $38^{\circ} \mathrm{C}$, a $39,1^{\circ} \mathrm{C}$ o estresse térmico está controlado e o animal não apresenta sinais de alteração no consumo de alimento, reprodução e produção. Temperatura interna (UTI) acima de $39,1^{\circ} \mathrm{C}$ ocorrem início do estresse térmico com sinais aparentes apenas no consumo de alimento, mas a reprodução e a produção permanecem estáveis. Acima de $40,1^{\circ} \mathrm{C}$ o estresse é evidente e os sinais clínicos são: Animal ofegante (variando de 100 a 120 movimento ruminal/min), redução drástica no consumo de alimento (consequentemente queda na produção), apresentação do estro diminuem, grandes reduções na produção, o consumo de alimento diminui $50 \%$ e a reprodução pode cair para $12 \%$, os animais expõem a língua e salivam muito, o animal fica apático impossibilitado de beber água e de se alimentar, podendo chegar a óbito.

Almeida et al. (2013) concluíram em seu trabalho que o tempo de arrefecimento dos animais no curral de espera, por 10, 20 e $30 \mathrm{~min}$, não proporcionaram alterações significativas na composição química e qualidade do leite, quando comparadas com as vacas que não foram submetidas ao sistema de climatização.

Conforme descrito na Tabela 1 não houve diferença significativa na avaliação da CCS nos meses de maio a setembro, isto indica que a temperatura interna do animal UTI, não influencia na CCS no leite entre as temperaturas avaliadas.

Tabela 1. Médias de CCS dos meses de maio a setembro nas temperaturas internas dos animais de $38^{\circ}$ a $39,5^{\circ}$

\begin{tabular}{lccccc}
\hline \multirow{2}{*}{$\begin{array}{l}\text { Temperatura } \\
\text { retal }\end{array}$} & \multicolumn{5}{c}{ Meses } \\
\cline { 2 - 6 } & Maio & Jun. & Jul. & Ago. & Set. \\
\hline $38^{\circ}$ & $180 \mathrm{Aa}$ & $102,4 \mathrm{Aa}$ & $170 \mathrm{a}$ & $113,8 \mathrm{Aa}$ & $106,6 \mathrm{Aa}$ \\
$8,5^{\circ}$ & $198,8 \mathrm{Aa}$ & $114 \mathrm{Aa}$ & $130 \mathrm{Aa}$ & $184,4 \mathrm{Aa}$ & $114,6 \mathrm{Aa}$ \\
$39^{\circ}$ & $213 \mathrm{Aa}$ & $170 \mathrm{Aa}$ & $119,4 \mathrm{Aa}$ & $116,6 \mathrm{Aa}$ & $97,0 \mathrm{Aa}$ \\
$39,5^{\circ}$ & $125 \mathrm{Aa}$ & $182,6 \mathrm{Aa}$ & $122,8 \mathrm{Aa}$ & $160,2 \mathrm{Aa}$ & $141,4 \mathrm{Aa}$ \\
\hline
\end{tabular}

Médias seguidas pela mesma letra maiúscula na linha e minúscula na coluna, não diferem estatisticamente entre si. Foi aplicado o Teste de Tukey ao nível de 5\% de probabilidade.

Barbosa et al. (2004) afirmam que o calor não influência na infecção em nem promove aumento da CCS em úberes não infectados, pois a CCS está relacionada com manejo sanitário. Conforme descrito na Tabela 2, não houve diferença significativa na avaliação da proteína nos meses de maio a setembro, isto indica que a temperatura interna do animal UTI, não influencia na composição da proteína.

Tabela 2. Médias de PB dos meses de maio a setembro nas temperaturas internas dos animais de $38^{\circ}$ a $39,5^{\circ}$.

\begin{tabular}{lccccc}
\hline \multirow{2}{*}{$\begin{array}{l}\text { Temperatura } \\
\text { retal }\end{array}$} & \multicolumn{5}{c}{ Meses } \\
\cline { 2 - 6 } & Maio & Jun. & Jul. & Ago. & Set. \\
\hline $38^{\circ}$ & $3,36 \mathrm{Aa}$ & $2,72 \mathrm{Aa}$ & $2.78 \mathrm{Aa}$ & $3.032 \mathrm{Aa}$ & $3.23 \mathrm{Aa}$ \\
$8,5^{\circ}$ & $2,91 \mathrm{Aa}$ & $2,85 \mathrm{Aa}$ & $3.01 \mathrm{Aa}$ & $3.13 \mathrm{Aa}$ & $3.19 \mathrm{Aa}$ \\
$39^{\circ}$ & $3,55 \mathrm{Aa}$ & $2,64 \mathrm{ABa}$ & $2.97 \mathrm{Aa}$ & $2.89 \mathrm{Aa}$ & $3.14 \mathrm{Aa}$ \\
$39,5^{\circ}$ & $2,88 \mathrm{Aa}$ & $3,03 \mathrm{Aa}$ & $3.10 \mathrm{Aa}$ & $2.96 \mathrm{Aa}$ & $3.12 \mathrm{Aa}$ \\
\hline
\end{tabular}

Médias seguidas pela mesma letra maiúscula na linha $\mathrm{e}$ minúscula na coluna, não diferem estatisticamente entre si. Foi aplicado o Teste de Tukey ao nível de 5\% de probabilidade.

Barbosa et al. (2004) relatam que os animais expostos ao sol, quando receberam água sob aspersão, mostraram maiores valores do teor de gordura e de proteína do leite, e o inverso ocorreu com os animais à sombra, em que o aumento no teor de gordura e de proteína foi observado quando a aspersão não ocorreu. Podemos observar que qualquer alteração relacionada com a diminuição do consumo de alimentos resulta na queda da qualidade do leite.

Porcionato et al. (2009) afirmam que para cada situação existe uma alternativa eficiente para reduzir os efeitos negativos do estresse térmico sobre a produção e qualidade do leite e sobre o bem-estar animal. Assim, devem se lançar mão de 
alternativas que buscam aumentar o conforto térmico dos animais, considerando as características climáticas relativas a cada propriedade e a região onde se localiza, além das características do rebanho e relação custo benefício.

Conforme descrito na Tabela 3 , não houve diferença na avaliação da gordura nos meses de maio a setembro, isto indica que a temperatura interna do animal UTI, não influencia na composição da gordura.

Nääs \& Arcaro Júnior (2001) ao avaliarem acondicionamento térmico para vacas leiteiras em condições de calor, perceberam que a porcentagem de gordura não apresentou diferenças significativas, mostrando o benefício do arrefecimento e contribuindo para o conforto dos animais.

Tabela 3. Médias de gordura no leite dos meses de maio a setembro nas temperaturas interna doa animais de $38^{\circ}$ a $39,5^{\circ}$.

\begin{tabular}{lccrcr}
\hline \multirow{2}{*}{$\begin{array}{l}\text { Temperatura } \\
\text { retal }\end{array}$} & \multicolumn{5}{c}{ Meses } \\
\cline { 2 - 6 } & Maio & Jun. & Jul. & Ago. & Set. \\
\hline $38^{\circ}$ & $2,70 \mathrm{Aa}$ & $3,49 \mathrm{Aa}$ & $2,68 \mathrm{Aa}$ & $2,75 \mathrm{Aa}$ & $2,54 \mathrm{Aa}$ \\
$8,5^{\circ}$ & $2,86 \mathrm{Aa}$ & $2,80 \mathrm{Aa}$ & $2,30 \mathrm{Aa}$ & $2,59 \mathrm{Aa}$ & $2,96 \mathrm{Aa}$ \\
$39^{\circ}$ & $3,03 \mathrm{Aa}$ & $2,49 \mathrm{Aa}$ & $3,28 \mathrm{Aa}$ & $2,64 \mathrm{Aa}$ & $2,28 \mathrm{Aa}$ \\
$39,5^{\circ}$ & $3,23 \mathrm{Aa}$ & $3,84 \mathrm{Aa}$ & $1,80 \mathrm{Aa}$ & $2,63 \mathrm{Aa}$ & $3,26 \mathrm{Aa}$ \\
\hline
\end{tabular}

As médias seguidas pela mesma letra não diferem estatisticamente entre si. Foi aplicado o Teste de Tukey ao nível de $5 \%$ de probabilidade.

Os resultados analisados da produção mostrados na tabela 4, indicam que mesmo em climas de mais calor como o de Goianésia quando os animais são mantidos em condições de sombra fornecimento adequado de água os animais mantêm a sua produção mostrando que não sofreu interferência do ambiente nestas condições.

Tabela 4. Médias de produção de leite dos meses de maio a setembro nas temperaturas interna dos animais de $38^{\circ}$ a $39,5^{\circ}$

\begin{tabular}{lccccc}
\hline \multirow{2}{*}{$\begin{array}{l}\text { Temperatura } \\
\text { retal }\end{array}$} & \multicolumn{5}{c}{ Meses } \\
\cline { 2 - 6 } & Maio & Jun. & Jul. & Ago & Set. \\
\hline $38^{\circ}$ & 14,46 & 16,6 & 17,6 & 18,0 & 17,5 \\
$8,5^{\circ}$ & 15,4 & 15,4 & 15 & 15,8 & 17,6 \\
$39^{\circ}$ & 18,0 & 18,2 & 19,4 & 18,8 & 17,6 \\
$39,5^{\circ}$ & 18,6 & 20,5 & 21 & 21,8 & 21,9 \\
\hline
\end{tabular}

Rodrigues et al. (2010) afirmam que em locais de alta temperatura, a principal resposta animal é a diminuição da produção de calor metabólico, obtida por redução no consumo de alimentos, o que consequentemente causa perdas na produção de leite.
Almeida et al. (2013) relatam que a exposição dos animais à climatização por 30 min no curral de espera apresentaram maior frequência de acesso ao comedouro, bebedouro e tempo de ruminação, indicando baixo nível de estresse e melhoria no bem-estar animal, com maior produção de leite.

\section{Conclusão}

Em ambiente de domínio cerrado nos meses de maio a setembro é possível obter resultados satisfatórios na produção e qualidade do leite, desde que se invista em áreas sombreadas e com água de fácil acesso aos animais, tornando o ambiente mais confortável. Foi observado que nestas condições não ocorre diferenças estatísticas na qualidade do leite, nem redução na produção de leite neste mesmo período.

\section{Referências Bibliográficas}

Almeida, G. L. P., Pandorfi, H., Barbosa, S. B. P., Pereira, D. F., Guiselini, C. \& De Almeida, G. A. P. 2013. Comportamento, produção e qualidade do leite de vacas Holandês-Gir com climatização no curral. Revista Brasileira de Engenharia Agrícola e Ambiental, 17, 892899.

Arcaro Junior, I., Arcaro, J. R. P., Pozzi, C. R., Fagundes, H., Matarazzo, S. V. \& Oliveira, C. A. 2003. Plasmatic levels of hormones, production and composition of milk in an acclamatized waiting room. Revista Brasileira de Engenharia Agrícola e Ambiental, 7, 350354.

Barbosa, O. R., Boza, P. R., Santos, G. T., Sakagushi, E. S. \& Ribas, N. P. 2004. Efeitos da sombra e da aspersão de água na produção de leite de vacas da raça Holandesa durante o verão. Acta Scientiarum. Animal Sciences, 26, 115-122.

Craesmeyer, K. C., Schmitt Filho, A. L., Hotzel, M. J., Deniz, M. \& Farley, J. 2017. Utilização da sombra por vacas lactantes sob sistema voisin silvipastoril no sul do Brasil. Cadernos de Agroecologia, 11, 1-6.

Head, H. H. 1996. Manejo de animais em sistema de estabulação livre visando maximizar conforto e produção. In: Moura, A. C. (ed.) Congresso Brasileiro de Gado Leiteiro Conceitos Modernos de Exploração Leiteira. FEALQ, Piracicaba.

INMET - Instituto nacional de Meteorologia. 2017. Precipitação Total Anual. Disponível 
em:

http://www.inmet.gov.br/portal/index.php?r=c lima/normaisClimatologicas.

Köppen, W. \& Geiger, R. 1928. Klimate der Erde. Gotha: Verlag Justus Perthes. Wall-map $150 \mathrm{~cm} x 200 \mathrm{~cm}$.

Nääs, I. A. \& Arcaro Júnior, I. 2001. Influência de ventilação e aspersão em sistemas de sombreamento artificial para vacas em lactação em condições de calor. Revista Brasileira de Engenharia Agrícola e Ambiental, 5, 139-142.

Pires, M. F. t. A. v. \& Campos, A. s. T. 2004. Modificações ambientais para reduzir o estresse calórico em gado de leite. EMBRAPA, $1,1-6$.

Porcionato, M. A. F., Fernandes, A. M., Netto, A. S. \& dos Santos, M. V. 2009. Influência do estresse calórico na produção e qualidade do leite. Revista Acadêmica: Ciência Animal, 7, 483-490.
Rezende, S. R., Munhoz, S. K., Nascimento, M. R. B. M. \& Guimarães, J. L. N. 2016. Características de termorregulação em vacas leiteiras em ambiente tropical: revisão. Veterinária Notícias, 21, 18-29.

Rodrigues, A. L., Souza, B. B. \& Pereira Filho, J. M. 2010. Influência do sombreamento e dos sistemas de resfriamento no conforto térmico de vacas leiteiras. Agropecuária Científica no Semiárido, 6, 14-22.

\section{Article History:}

Received 3 January 2018

Accepted 17 February 2018

Available online 16 April 2018

License information: This is an open-access article distributed under the terms of the Creative Commons Attribution License 4.0, which permits unrestricted use, distribution, and reproduction in any medium, provided the original work is properly cited. 